\title{
Visually detected NREM Stage 2 sleep spindles in kindergarten children are associated with stress challenge and coping strategies
}

\author{
THORSTEN MIKOTEIT ${ }^{1}$, SERGE BRAND 2 , JOHANNES BECK ${ }^{2}$, SONJA PERREN ${ }^{3}$, \\ AGNES VON WYL ${ }^{4}$, KAI VON KLITZING ${ }^{5}$, EDITH HOLSBOER-TRACHSLER ${ }^{2}$ \\ \& MARTIN HATZINGER ${ }^{1,6}$
}

\begin{abstract}
${ }^{1}$ Psychiatric Hospital of the University of Basel, Psychiatric Out-Patient Clinic, Basel, Switzerland, ${ }^{2}$ Psychiatric Hospital of the University of Basel, Depression and Sleep Research Unit, Basel, Switzerland, ${ }^{3}$ University of Zurich, Facobs Center for Productive Youth Development, Zurich, Switzerland, ${ }^{4}$ University of Applied Science Zurich (ZHAW), Department of Applied Psychology, Zurich, Switzerland, ${ }^{5}$ University of Leipzig, Department of Child and Adolescent Psychiatry, Psychotherapy, and Psychosomatics, Leipzig, Germany, and ${ }^{6}$ Psychiatric Services Solothurn, Department of Adult Psychiatry, Solothurn, Switzerland
\end{abstract}

\begin{abstract}
Objectives. Sleep EEG spindles are linked to efficient cortical-subcortical connectivity and intellectual abilities. The aim of the present study was to investigate the relationship of spindle activity to psychosocial stress response and coping strategies in healthy kindergarten children. Methods. In a cross-sectional study of 415 -year old kindergarten children we examined stress-induced hypothalamic-pituitary-adrenocortical (HPA) system activity by saliva cortisol measurements and sleep regulation by sleep EEG-monitoring. Stress response was measured during the application of a standardized psychological challenge appropriate at this age. NREM S2 sleep EEG spindles were visually scored and put into relation to coping and HPA activity parameters. Results. An increased total number of NREM S2 sleep spindles correlated positively with increased high ego-involvement strategies such as "positive emotions". By contrast, total number of NREM S2 sleep spindles correlated negatively with low ego-involvement strategies such as "denial" and "avoidance". Stress induced HPA-activity correlated positively with coping strategies with high ego-involvement; while there was no correlation with low ego-involvement strategies. Conclusions. Total number of visually detected NREM S2 sleep spindles is elevated in children with coping involving positive, high ego-involvement; in contrast, low ego-involvement during stress is associated with reduced total number of NREM S2 sleep spindles.
\end{abstract}

Key words: Sleep spindles, polysomnography, kindergarten children, stress challenge, free saliva cortisol

\section{Introduction}

Kindergarten children are characteristically encountered by the demand of developing social skills and learning to cope with psychosocial stress challenges. These developments depend on intact learning and memory functioning, which involve complex neurobiological processes. Sleep may reflect developmental stages as well as the individual capability to learn and cope with stress and adaptation. There is striking evidence that sleep and learning are related: Recent research has focused on memory consolidation during sleep (Stickgold 2005; Stickgold and Walker 2007). Consolidation of declarative memory, which is already evident in children, has been linked to
NREM sleep, especially to slow wave sleep (SWS) and sleep spindles (Plihal and Born 1997; Gais et al. 2002; Schabus et al. 2004; Fischer et al. 2007; Wilhelm et al. 2008; Genzel et al. 2009; Born 2010; for extensive overview, see Diekelmann and Born 2010).

Sleep spindles, which characteristically occur in NREM stage 2 sleep, are spindle like, rhythmic EEG-oscillations in a frequency range between 11 and $14.75 \mathrm{~Hz}$ lasting from 0.5 to $3 \mathrm{~s}$ (De Gennaro and Ferrara 2003; Grigg-Damberger et al. 2007). Sleep spindles have been linked to efficient corticalsubcortical connectivity and they are positively correlated with cognitive and learning abilities (Schabus et al.

Correspondence: Dr. med. Thorsten Mikoteit, Psychiatric Hospital of the University of Basel, Psychiatric Out-Patient Clinic, Petersgraben 4, 4031 Basel, Switzerland. Tel: +41612655040 . Fax: +41 61 265 4588. E-mail: thorsten.mikoteit@upkbs.ch 
2004, 2006, 2008; Bódizs et al. 2005; Fogel and Smith 2006; Fogel et al. 2007). However, there is a complete lack of knowledge with respect to the relation between spindle activity and cognitive processes such as coping with challenging situations in preschoolers.

In recent years, considerable research has focused on the linkage between sleep and the physiological stress response, both of which are regulated by the HPA axis system (Steiger 2002, 2007). Alterations in HPA-activity are consistently correlated with changes in sleep structure, e.g., REM-sleep distribution (Hatzinger et al. 2002, 2004). The relationship of HPA-functioning with NREM-sleep parameters like sleep spindles or SWS are less consistent. In kindergarten children HPA-hyperactivity is related to poor sleep patterns and more sleep in NREMstage 1 and 2 (Hatzinger et al. 2008, 2010). So far, there is poor evidence on correlations between HPA-activity and sleep spindles.

In kindergarten children, increased HPA system activity was significantly associated with behavioural/ emotional difficulties like internalizing problems (Lopez et al. 2004) or hyperactivity/impulsivity in boys (Hatzinger et al. 2007). Hypoactivity of HPA system was associated with hostility, antisocial behavior (Flinn and England 1997), aggression and oppositional behaviour (van Goozen et al. 1998; Shirtcliff et al. 2005; El-Sheikh et al. 2008). Moreover, increased cortisol secretion under challenge conditions is related to better cognitive performance (Blair et al. 2005) and positive emotions in girls (Hatzinger et al. 2007); however, it is also related to behavioural difficulties in boys (Flinn and England 1997; Hatzinger et al. 2007; El-Sheikh et al. 2008).

The aim of this explorative study was to investigate the interactions between NREM S2 sleep spindles, stress response and cognitive functioning under stress challenges in kindergarten children. We formulated the following hypotheses: (1) we anticipated that cognitive processes such as mastering challenging social situations should be related to NREM S2 sleep spindles; (2) increased salivary cortisol secretion under challenging conditions is associated with both favourable and unfavourable cognitive processes; (3) finally, we explored whether the reactivity of HPA-axis under challenge conditions was related to NREM S2 sleep spindles.

\section{Method}

\section{Study population}

One hundred and two children at the age of $4.91 \pm$ 0.44 (mean \pm SD) years starting with kindergarten were recruited from 18 kindergartens in an urban area of Switzerland (see also Perren et al. 2006; Hatzinger et al. 2007). The kindergarten classes were selected from different city districts representing various socioeconomic and ethnic backgrounds representative for an urban area in Switzerland. The children were enrolled in this exploratory, crosssectional study after a thorough clinical examination to exclude any relevant medical and/or neurological disorders. Parents and children were thoroughly interviewed about the children's present and past physical and mental health, as well as about use of any psychotropic substances. We established that all participants could normally sleep in their bed without interruptions due to travelling, noise, siblings or illness. Furthermore, none of the participants had been subjected to sleep deprivation, time shifts or intake of any disturbing substances/medication (e.g., stimulants) during the 3 months prior to the investigation. From these 102 children, 67 (35 boys and 32 girls) agreed to undergo EEG sleep profile analysis. Of a subsample of 41 children, who rendered complete data sets, EEG-sleep recordings were analyzed for NREM S2 sleep spindles. The children of this sample (mean age 5.34 $\pm 0.34,20$ boys and 21 girls) did not differ from the initial sample $(N=67)$ with respect to age, gender, cortisol secretion and behavioural/emotional conditions.

\section{Procedure}

All children were interviewed individually in a separate room in their kindergarten or at home (depending on the recruitment scheme) by a trained psychologist. The standardized assessment battery included the Berkley Puppet Interview (BPI; Measelle et al. 1998) and the MacArthur Story Stem Battery (MSSB; Von Klitzing et al. 2003; Stadelmann 2006). Cortisol samplings were performed during the MSSB-task. Teachers and parents completed questionnaires. Within 4 weeks after the psychological assessment, children's sleep was assessed at their home. With respect to ethical considerations, the purpose and experimental details of the study were fully explained to the children and their parents. The parents were asked to sign an informed consent form before their children entered the study. Additionally, the experimental protocol was carried out in accordance with the Declaration of Helsinki and it was approved by the local ethical committee.

\section{Instruments}

Children's coping strategies. To assess coping strategies, the MSSB was applied, which was extensively described elsewhere (Von Klitzing et al. 2003; Stadelmann 2006). In MSSB children had to continue 
and/or finish a presented story stem which - like in the Trier Social Stress Test for Children (TSST-C) demands high ego-involvement. In detail, the MSSB uses standardized, developmentally appropriate beginnings of stories to elicit relevant play narratives. A well experienced investigator administers eight MSSB story stems to each of the children by using little play figures. The stories contain stressful events from children's everyday life, like loss of a family's pet, quarrel between parents, or a moral dilemma. The story stems built up to a dramatic high point of conflict, and the child is then asked to show and tell what happens next in front of the unknown investigator and the video camera. The play narratives were videotaped and coded with the MacArthur Narrative Coding system (Robinson et al. 2002). Narrative content (dysregulated aggression, negative and positive emotions, affectional themes, limit setting, positive and negative parental representations) and narrative performance (coherence, embellishment and avoidant strategies) were rated (see Von Klitzing et al. 2003).

Self report of difficulties/competences. To assess children's difficulties and competences, the BPI (Measelle et al. 1998) was applied. The BPI is carried out by means of two identical hand puppets that make two opposing statements on a topic, following which the child can give his/her own statement. The interview is videotaped and subsequently scored by independent raters. Each item is rated on a seven-point scale. The following scales were assessed: depression, separation anxiety, over-anxiousness, oppositionality/ defiance, overt aggression to peers, impulsivity, pro-social behaviour and peer victimization (i.e. being the target of peer aggression or bullying).

Sleep assessment. Sleep was recorded with an ambulatory EEG system (Oxford-Medilog 9200). EEG was registered from $\mathrm{C} 3-\mathrm{A} 2$ and $\mathrm{C} 4-\mathrm{A} 1$. For electromyogram two electrodes were put on the chin. Electrooculogram was registered by two electrodes on the right and left side. A1 and A2 electrodes were physically linked. Sensitivity for input-measurement was $200 \mu \mathrm{V}$ peak to peak $( \pm 100 \mu \mathrm{V})$ for EEG and $50 \mu \mathrm{V}$ peak to peak $( \pm 25 \mu \mathrm{V})$ for $\mathrm{EMG}$ and EOG. Sleep was recorded during two consecutive nights at the children's home. Electrodes for polysomnographic recordings were fixed the evening before registration and removed the next morning. The first adaptation night served to familiarize the children with the recording, including attachment of the electrodes. Only data from the second night were assessed for computations. For that, we assume that so called first night effects did not systematically bias the present data. Parents were instructed to report any deviation of sleep routine and any major stress events which might have affected children's sleep. Sleep recordings were neither performed at the time of holidays, Christmas, birthdays, other family events nor during sportive or cultural local festivities. Sleep EEGs were visually analyzed by two highly experienced raters according to the standard procedures (Rechtschaffen and Kales 1968). Sleep parameters were analyzed according to the definitions in the standard program described by Lauer et al. (1991). The raters' overall interrater reliability was $r=0.92$ (Brand et al. 2010).

Sleep spindles were visually scored in NREMStage 2 (S 2). Typical spindle morphology and a minimum duration of $0.5 \mathrm{~s}$ were the main criteria to detect a spindle. Most spindles were in the frequency range of about $12-14 \mathrm{~Hz}$, however spindles of lower or higher frequency were also included. NREM S2 spindle density was calculated as the ratio of the number of NREM S2 sleep spindles per hour of NREM S2 sleep.

HPA-system assessment under challenge conditions. Since for preschool-children a non-pharmacological stress test demanding a high ego involvement and leading to a reliable and reproducible hormonal response is lacking, the MSSB was used. According to an adapted TSST-C protocol cortisol samplings were performed during the MSSB-task as described above. MSSB tests were conducted in the kindergarten on a fixed schedule exclusively during late morning, i.e. between 10.00 and $11.00 \mathrm{~h}$. In order to reach a standardized contextual baseline, children were familiarized with the situation by the initial contact with the investigator conducting a short pleasant activity such as playing an easy card game, building a tower with wooden pieces and applying a short vocabulary test for distraction (Peabody Picture Vocabulary Test; Dunn and Dunn 1981). During this time frame the first two salivary cortisol samplings were collected with taking the first one $10 \mathrm{~min}$ and the second one $1 \mathrm{~min}$ before starting the MSSB-task. The third (20 min after the MSSB start) and the fourth ( $35 \mathrm{~min}$ after the MSSB start) samples were taken during and at the end of the MSSB-task. To calculate the cortisol release as a function of the MSSB-task, the area-under-theconcentration-time-curve (AUC, arbitrary units; see also below) was calculated integrating the second, third, and fourth saliva samples. After a recovery time of about $10 \mathrm{~min}$ the fifth and last MSSB-related saliva sample was collected. Subsequently, further tests including the Berkley Puppet Interview (BPI) were conducted. 
Saliva cortisol sampling technique and cortisol analysis. Saliva samples were obtained using the "Salivette" device for quick and hygienic sampling (Sarstedt, Nümbrecht/Germany). This device mainly consisted of a small cotton swab on which the subject gently chewed for $0.5-1 \mathrm{~min}$. Thereafter, the swab was transferred into a small plastic tube, the "Salivette" container, and stored in the freezer. Saliva samples were returned to the laboratory, where samples were centrifuged at $4^{\circ} \mathrm{C}(2000 \mathrm{rpm}, 10 \mathrm{~min})$ and stored at $-20^{\circ} \mathrm{C}$ until assay. Free salivary cortisol concentrations were analyzed using a time-resolved immunoassay with fluorometric detection "Coat-A-Count" Cortisol RIA from DPC (Diagnostics Products Corporation; obtained through $\mathrm{H}$. Biermann $\mathrm{GmbH}$, Bad Nauheim, Germany) as described in detail elsewhere (Dressendörfer et al. 1992; Tunn et al. 1992). Intra- and interassay variability of this assay was less than 4.31 and $5.20 \%$, respectively.

\section{Statistical analyses}

For correlations, Pearson's $r$ was calculated. Test results with an alpha level of below 0.05 are reported as significant. In the present research we did choose an explorative approach; for this reason, no alpha-correction for multiple testing was introduced, because following Harris et al. (2009) overcontrolling for type I error rate would have made less sense.

To indicate the hormone release of cortisol samples during the challenge test (MSSB) the areaunder-the-concentration-time-curve between the second and fourth sample (AUC, arbitrary units) was computed by using the trapezoidal integration (Forsythe et al. 1969).

Statistical analyses were performed using SPSS 17.0 for Windows.

\section{Results}

\section{Sleep-EEG results}

Variables of sleep continuity, sleep stages, REMsleep variables and NREM S2 spindle results are depicted in Table $\mathrm{I}$.

\section{Visually detected NREM S2 sleep spindles and} cognitive processes under challenge conditions

We could demonstrate several associations of visually detected NREM S2 sleep spindles with behavioural/ psychological variables (Table II): Total number of NREM S2 sleep spindles is positive, correlated with "positive emotions" in MSSB $(r=0.50 ; P=0.001)$ and with "prosocial behaviour" in BPI $(r=0.36$;
Table I. Descriptive overview of EEG-sleep variables of the study population $(n=41)$.

\begin{tabular}{|c|c|}
\hline & Mean (SD) \\
\hline \multicolumn{2}{|l|}{ Sleep continutity variables } \\
\hline $\begin{array}{l}\text { Total sleep time (TST) } \\
\text { (min) }\end{array}$ & $608.50(40.57)$ \\
\hline $\begin{array}{l}\text { Sleep period time }(\mathrm{SPT}) \\
(\mathrm{min})\end{array}$ & $643.15(40.61)$ \\
\hline $\begin{array}{l}\text { Sleep efficiency index (SEI) } \\
(\%)\end{array}$ & $96.16(2.84)$ \\
\hline $\begin{array}{l}\text { Sleep onset latency (SOL) } \\
\text { (min) }\end{array}$ & $11.79(11.90)$ \\
\hline $\begin{array}{l}\text { Waketime after sleep onset } \\
\text { (WASO) (min) }\end{array}$ & $22.08(16.26)$ \\
\hline \multicolumn{2}{|l|}{ Sleep architecture } \\
\hline NREM Stage $1(\mathrm{~min})(\%)$ & $7.19(5.90) 1.18(0.97)$ \\
\hline Stage $2(\min )(\%)$ & $201.20(68.23) 33.03(11.21)$ \\
\hline Stage $3(\min )(\%)$ & $98.81(48.15) 16.24(7.91)$ \\
\hline Stage $4(\min )(\%)$ & $181.70(48.99) 29.86(8.05)$ \\
\hline $\begin{array}{l}\text { SWS-time }(\mathrm{S} 3+\mathrm{S} 4) \\
\quad(\min )(\%)\end{array}$ & $280.51(69.02) 46.08(11.34)$ \\
\hline \multicolumn{2}{|l|}{ REM sleep } \\
\hline REM-time ( $\mathrm{min}$ ) & $119.80(30.86)$ \\
\hline$(\%)$ & $19.69(5.07)$ \\
\hline REM-latency (min) & $126.15(54.64)$ \\
\hline REM-density & $3.45(0.89)$ \\
\hline \multicolumn{2}{|l|}{ NREM S2 sleep spindles } \\
\hline $\begin{array}{l}\text { Total number of NREM S2 } \\
\text { sleep spindles }(n)\end{array}$ & $396.36(251.75)$ \\
\hline $\begin{array}{l}\text { NREM } S 2 \text { sleep spindle } \\
\text { density }(\mathrm{n} / \mathrm{h})\end{array}$ & $119.75(78.59)$ \\
\hline
\end{tabular}

REM-density = the ratio of 3-s mini-epochs per REM period including at least one rapid eye movement to the total amount of all 3-S mini-epochs per REM period.

$P=0.022)$. No correlations were found between the total number of NREM S2 sleep spindles and the dimensions "embellishment", "coherence", "dysregulated aggression", "limit setting", "positive" and "negative parental representations" (MSSB), and "depression" (BPI). ${ }^{1}$ There were negative correlations between total number of NREM S2 sleep spindles and "denial" $(r=-0.40 ; P=0.009$, Figure 1), "avoidant strategies" $(r=-0.31 ; P=0.05)$ and "impulsivity" ( $r=-0.34 ; P=0.034$ ). Separate correlational computations revealed that "denial" did positively correlate with S3 $(r=0.32, P<0.05)$; "positive emotions" did positively correlate with $S 1$ $(r=0.50, P<0.01$ ), whereas "prosocial behavior" did not correlate with any dimension of sleep continuity and sleep architecture.

\footnotetext{
'To further test, if and to which extent avoidance, denial, positive emotion (MSSB) and prosocial behavior and impulsivity (BPI) did correlate with the mere length of NREM S2 sleep and NREM S2 sleep spindle density respectively, correlations were performed. Except for impulsivity $(r=-0.40, P<0.05)$, all other correlation coefficients were $<0.30$ and did not reach statistical significance.
} 
Table II. Correlations of emotional and behavioural items with visually detected total number of NREM S2 sleep spindles and salivary cortisol under stress challenge.

\begin{tabular}{|c|c|c|}
\hline & $\begin{array}{c}\text { Stress } \\
\text { cortisol } \\
\text { (AUC total) } \\
\text { (correlation } r \text { ) }\end{array}$ & $\begin{array}{l}\text { Total number } \\
\text { of NREM S2 } \\
\text { sleep spindles } \\
\text { (correlation } r \text { ) }\end{array}$ \\
\hline \multicolumn{3}{|l|}{ MSSB } \\
\hline \multicolumn{3}{|l|}{ dysregulated aggression } \\
\hline $\begin{array}{l}\text { avoidant strategies } \\
\text { embellishement }\end{array}$ & & $-0.308^{z}$ \\
\hline denial & & $-0.403^{* *}$ \\
\hline positive emotions & $0.586^{\text {w* }}$ & $0.498^{* *}$ \\
\hline \multicolumn{3}{|l|}{$\begin{array}{l}\text { parental representations } \\
\text { negative }\end{array}$} \\
\hline $\begin{array}{l}\text { parental representations } \\
\text { discipline }\end{array}$ & $0.355^{4}$ & \\
\hline \multicolumn{2}{|l|}{$\begin{array}{l}\text { parental representations } \\
\text { positive }\end{array}$} & \\
\hline \multicolumn{3}{|l|}{$\mathrm{BPI}$} \\
\hline \multicolumn{3}{|l|}{ depression } \\
\hline impulsivity & & $-0.335^{*}$ \\
\hline prosocial behavior & & $0.356^{2}$ \\
\hline
\end{tabular}

MSSB, MacArthur Story Stem Battery; BPI, Berkeley Puppet Interview; AUC total, area under the cortisol-time curve between second and fourth salivary cortisol sample during MSSB. Pearson's correlations. ${ }^{*} P<0.05$ (two-tailed), ${ }^{* *} P<0.01$ (two-tailed). Only coefficients with a probability value of $P<0.05$ were presented.

In summary, in children showing an active and positive coping style total number of NREM S2 sleep spindles was increased, while in children showing a negative and avoidant coping style total number of NREM S2 sleep spindles was decreased. To further prove this pattern of results, the psychological dimensions of "Denial" and "Positive emotions" were median-split. Next, children were assigned to one of three categories: "Low denial and high positive emotions (LD/HPE; $n=11$ )"; "High denial and high positive emotions/low denial and low positive emotions (HD/HPE_LD/LPE; $n=15$ )", and "High denial and low positive emotions (HD/LPE; $n=15)$ ". The three groups did differ neither for

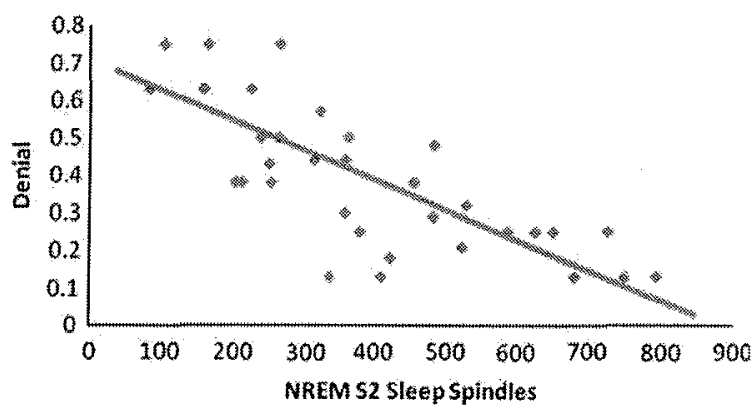

Figure 1. Increased total number of NREM S2 sleep spindles correlated with decreased denial $\left(r=-0.40^{* *}\right)$. age $(F(2,38)=2.20, P=0.12)$, nor for the length of NREM S2 sleep $(F(2,38)=1.34, P=0.27)$; therefore, age and length of NREM S2 sleep were not introduced as covariates. The ANOVA with the factor Categories and the dependent variable Total number of NREM S2 sleep spindles revealed significant mean differences $(F(2,38)=3.86$, $P=0.03 ; \mathrm{LD} / \mathrm{HPE}: M=545.55, \mathrm{SD}=403.77$; HD/HPE_LD/LPE: $M=313.67, \mathrm{SD}=161.85$; HD/LPE: $M=304.93, S D=123.86)$. A post-hoc analysis after Games-Howell revealed that the total number of NREM S2 sleep spindles was significantly statistically highest in the category with "Low denial and high positive emotions" compared to the other two categories.

\section{Stress-induced HPA-activity and coping}

An increased cortisol secretion was positively associated with "positive emotions" (AUC total MSSB: $r=0.59 ; P<0.001$; Table II; Figure 2). Children with high scores in MSSB item "positive emotions" showed higher salivary cortisol at time point 3, i.e. 20 min after MSSB-test started, than children with low scores (Figure $3 ; t(35)=2.40 ; P=0.022$ ). There were also positive correlations between stress induced cortisol (AUC total) and "limit setting" and "parental representations discipline". No correlations were observed between cortisol (AUC total) and the MSSB-items "avoidance", "denial" or "dysregulated aggression" (Table II). Stress-induced cortisol was not correlated with "prosocial behaviour" or any other BPI-item.

\section{Stress-induced HPA-activity and visually detected NREM S2 sleep spindles}

We found no correlation between NREM S2 sleep spindles (neither total number nor spindle density) and stress-induced cortisol values (all $r$ values $<0.1$ ) .

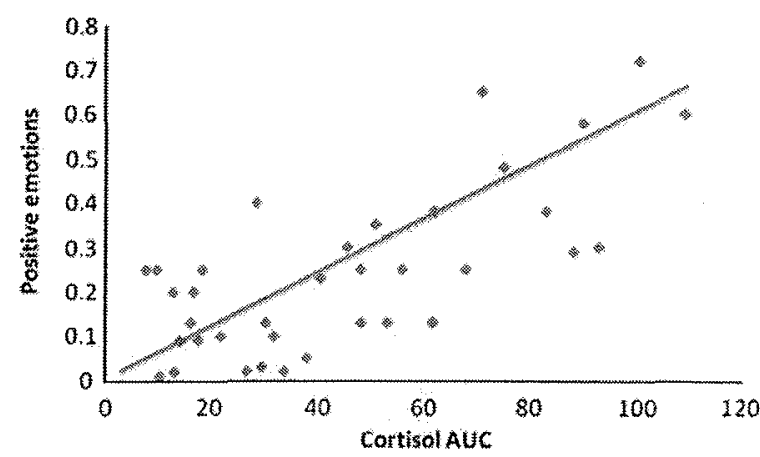

Figure 2. Increased cortisol secretion under stress conditions correlated with increased positive emotions $\left(r=0.59^{\text {t*s }}\right)$. 
Saliva cortisol and stress challenge

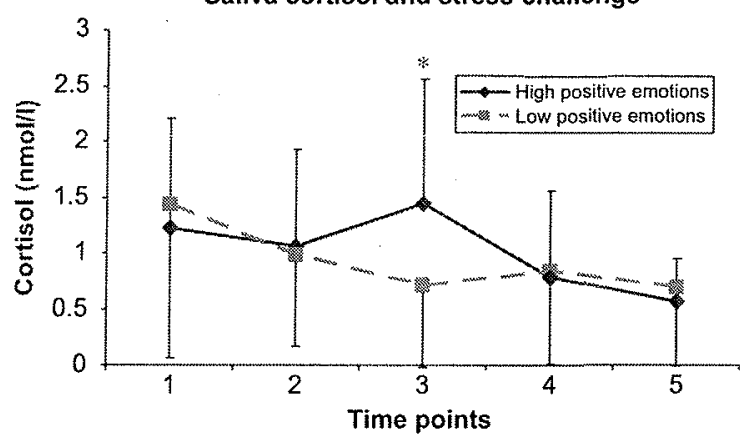

Figure 3. Comparison of salivary-cortisol-time-curve under stress challenge between children with high and low scores on MSSB item "positive emotions". Time points: $1=-10 \mathrm{~min} ; 2=-1 \mathrm{~min}$ $3=20 \mathrm{~min} ; 4=35 \mathrm{~min}$; in relation to start of MSSB test. Time point $5=10 \mathrm{~min}$ after MSSB challenge has finished. ${ }^{*} P<0.05$ ( $t$-test for independent samples).

\section{Discussion}

The key findings of the present study were that in 5-year-old children total number of NREM S2 sleep spindles varied as a function of cognitive strategies related to challenge, and that the cortisol secretion was related to both favourable and unfavourable cognitive processes. Two hypotheses were formulated and each of these is considered in turn.

First, we anticipated that cognitive processes such as mastering challenging social situations should be related to increased spindle activity, and the results confirmed this assumption: Increased total number of NREM S2 sleep spindles was associated with positive emotions and with prosocial behaviour. These coping strategies have in common a high degree of ego-involvement with active, positive and attentive attitudes. In contrast, unfavourable coping strategies with low ego-involvement such as avoidance or denial were associated with a decreased total number of NREM S2 sleep spindles. Therefore, the present results add to the literature insofar as increased spindle activity was associated with favorable coping, and not only with declarative memory consolidation (Schabus et al. 2008).

Second, we predicted an increased salivary cortisol secretion under challenge conditions both as a function of favourable and unfavourable cognitive processes, and, again, results confirmed the assumption: Coping strategies with high ego-involvement were related to increased cortisol levels. Importantly, high ego-involvement comprised completely opposite cognitive-emotional processes (positive emotions and parents perceived as having unfavourable parenting styles such as rigorous limit setting and discipline). Insofar, the present results add to the literature showing that a high egoinvolvement and an increased cortisol secretion are related both to favourable (e.g., Blair et al. 2005; Hatzinger et al. 2007) and unfavourable cognitiveemotional processes (e.g., Flinn and England 1997; Hatzinger et al. 2007).

In a dual-process model of the stress-response, there is an automatic process of response to stress (e.g., physiological and emotional arousal) and a second, voluntary part, which is termed coping. Coping has been defined as a subset of broader selfregulatory processes, which are volitionally and intentionally enacted especially in response to stress (Compas 2006). In children these coping strategies are connected to the biological, cognitive, social and emotional development in a bi-directional way: Coping depends on the resources of the child and its stage of development, while coping strategies have an impact on further development. Coping strategies can be distinguished as involving engagement with versus disengagement from sources of stress and one's emotional responses to stress. Engagement coping responses are further distinguished as primary control (or active) coping responses (problem solving, emotional expression, emotional modulation) and secondary control (or accommodative) coping (acceptance, cognitive restructuring, positive thinking, distraction). Disengagement coping includes avoidance, denial, and wishful thinking (ConnorSmith et al. 2000). Coping strategies are linked to temperament and depression in childhood. They may be an important mediator between children's temperament and its risk for developing affective disorders like depression (Compas et al. 2004).

The first explorative approach of our study focused on the relation between NREM S2 sleep spindles and cognitive (-emotional) processes, i.e. coping under stress challenge. According to our data, total number of NREM S2 sleep spindles is linked to stress-challenge in dependence of the involved coping strategy (i.e. positive emotion, denial and avoidance), whereas the mere length of NREM S2 sleep and NREM S2 sleep spindle density were not related to these psychological dimensions. Our results imply that engagement coping strategies both with high ego-involvement and with active, positive and attentive attitudes, which are useful for making new and favourable experiences, seem to be connected with an increased spindle activity. In contrast, unfavourable, disengaging coping strategies with low ego-involvement such as avoidance or denial, which decrease the possibility to make new experiences, are associated with a decreased spindle activity.

Though highly hypothetical, these results can be discussed with respect to the well known linkage between sleep spindles and memory consolidation: 
In the "off-line" reorganization and consolidation of declarative memory, containing facts and events, which could explicitly be retrieved, SWS and sleep spindles play a significant role (Marshall and Born 2007): Spindle activity is believed to facilitate neuroplastic cortical processes which are necessary for memory consolidation (Buzsáki 1996; Mölle and Born 2009). Therefore spindle activity might be a marker of declarative memory consolidation. In experimental studies Schabus et al. (2008) could demonstrate a learning dependent increase in spindle density (especially in the first half of the night). Spindle density correlates with memory consolidation and overnight improvement after a declarative memory task (Schabus et al. 2004, 2008). In children results of adults concerning declarative memory consolidation have been replicated (Fischer et al. 2007; Wilhelm et al. 2008).

We assume that children who use favourable, engagement coping strategies with high ego-involvement are more liable to make learning experiences. The resulting memory traces would be consolidated during NREM sleep which is reflected by increased total number of NREM S2 sleep spindles. However unfavourable, disengagement coping strategies with low ego-involvement, i.e. denial and avoidance, are strategies to avoid the elaboration and integration of unpleasant learning experiences. These coping strategies were negatively correlated with total number of NREM S2 sleep spindles. The results of MSSB coping strategies are paralleled by BPI items, which depict stress-independent traits. Children who are characterized by prosocial behaviour show higher total numbers of NREM S2 sleep spindles, while impulsivity is linked to low total number of NREM S2 sleep spindles.

Whether stress-challenges lead to learning experiences would mainly depend on coping strategies: Individuals who exert an engaging and active coping strategy during stress-situations are about to learn more easily than those who disengage. Therefore, we assume that emotional and behavioural coping during wakefulness will lead to learning and declarative memory processes which are reflected by spindle activity during sleep.

The second explorative approach referred to the relationship between increased cortisol levels under challenging conditions and coping strategies. Our results show that active coping strategies like "positive emotions" or "limit setting" were associated with increased cortisol secretion. While in contrast, disengaging strategies like "avoidance" or "denial" were not associated with stress-induced cortisol secretion. These results indicate that there is a linkage between psychological stress challenge in the MSSB, stress-induced cortisol, i.e. HPA-system activity and stress coping. As the MSSB rest is similar to the well evaluated Trier Social Stress Test for Children (TSST-C) these results can be discussed in reference to former studies with social stress-challenge studies in children: Children with coping strategies with high ego-involvement show increased cortisol release. This is in line with former reported results of studies by the TSST-C (e.g., Kirschbaum and Hellhammer 1989; Buske-Kirschbaum et al. 1997). Vice versa, HPA-activation seems to be associated with positive, engaged coping, and helps children to manage stress challenges in a socially favourable way. At the opposite, strategies with low ego-involvement like avoidance or denial were not correlated to stress-induced cortisol. These strategies seem to inhibit stress response. In contradiction to our expectations we found no correlation between dysregulated aggression or embellishment and stress-induced cortisol secretion. This may be due to the fact that association between stress-response and coping is only weak and therefore our sample was too small and heterogeneous to show an effect. Besides it is possible that negative, dysregulated coping may be an expression of an unsuccessful adaptation to a stress challenge to which activation of the HPA-system is an essential part.

Even though HPA axis activity is physiologically important to sleep regulation, HPA functioning as derived by stress elicited saliva cortisol in MSSB obviously does not have a direct impact on sleep spindle activity. From experimental studies it is known that a rise of cortisol level impairs hippocampusdependent declarative memory consolidation (Plihal and Born 1999; Wagner and Born 2008; Diekelmann and Born 2010). Therefore, one would expect that hyperactivity of HPA axis would result in reduced spindle activity. However NREM S2 sleep spindles seem to be independent from stress elicited HPA activity. Maybe the degree of stress-elicited cortisol which is a marker for ego-involvement and activation does not necessarily imply a general hypercortisolism during sleep. Moreover, HPA hyperactivity results mainly in alterations of REM-sleep in the second half of the night (Hatzinger et al. 2008). However, other mechanisms like the inhibition of acetylcholine which are more relevant to SWS and hippocampus-dependent memory consolidation than HPA-functioning may contribute to the present results (Hasselmo 2006).

Some limitations of our study warrant discussion. First, stress coping may essentially depend on the child's intellectual abilities, and this may be relevant for sleep dependent processes like memory consolidation. Fogel et al. (2007) demonstrated that spindle activity correlates with performance intelligence quotient (IQ). Unfortunately, we did neither 
perform cognitive testing, e.g., intelligence testing, nor any further neurodevelopmental examinations. Therefore, we cannot exclude that CNS maturity and intelligence are confounding the linkage between stress coping and spindle activity in our data. However, our non-clinical sample consisted of healthy kindergarten children; children with intellectual deficits were not included. Moreover, we postulate that intellectual abilities which are reflected by sleep spindles (Bódizs et al. 2005) will vice versa have an effect on stress coping at wake-time. In a further study it would be worthwhile to analyze whether IQ is a mediating factor between coping and sleep spindles.

Second, except for coping, variance of HPAactivity may be due to numerous other factors, e.g., time of day or exercise (Weitzman et al. 1971; Kanaley et al. 2001) and stress-elicited cortisol secretion may be affected by the varying basal cortisol level. However, both time of day and physical activity were rigorously controlled in our study. Therefore, a confounding contribution by these factors to our results seems unlikely.

Third, we cannot exclude that the lack of correlation between NREM S2 sleep spindle activity and stress induced HPA-activity reflects a false negative result: For technical reasons sleep EEG with assessment of spindle activity could not be performed immediately in the following night after MSSB stress test. Therefore we cannot exclude that we missed a weak effect. However, the focus of this paper is the relationship between the trait-like dimension of stress coping and spindle activity. The MacArthur Story Stem Battery (MSSB) is an experimental psychosocial stress challenge similar to other challenges children experience in everyday life. The MSSB does allow us to detect the children's coping style which is a trait-like feature. Therefore we assume that it may be reasonable to correlate children's characteristic stress coping to total number of NREM S2 sleep spindles, even if sleep recordings have not accomplished the consecutive night after the MSSB. We claim that HPA activity and stress coping consist of individually stable trait-like patterns, which are reflected in nocturnal sleep every day. Ziegert et al. (2001) showed that children's responses to challenging situations are stable over years. While this paper did not focus on the direct association between a particular stress challenge and spindle activity in the following night, it would be a good idea for a further study to test the direct effects of acute stress on sleep spindle activity in a stresscontrolled test-retest design.

Finally, the method of our ambulatory sleep-EEG recording and the algorithm of spindle detection have several limitations. For the aim of a population and home based study we used a simple but robust recording two-EEG-channel device. Our analysis relied on traditionally used central sites of EEG derivations (C3, C4). As we did not differentiate between slow frontal spindles and fast posterior spindles, the traditionally used central sites of EEG derivations may combine information of both spindle sites. Indeed, Schabus et al. (2006) could show that results on spindle activity and general learning abilities were most robust at central sites, whereas frontopolar and occipital sites did not add further information. Besides, centroparietal spindles seem to be less sensitive to CNS maturation and change little by age (Shinomiya et al. 1999). Therefore, without differentiation of spindle types, in this pilot study C3, C4 seems to be the appropriate sites of spindle detection. Unfortunately, our EEG recording procedures did not allow us to do EEG-frequency analyses nor assessments of EEG power distribution. Besides because of our visual spindle detection method, we were not able to assess further variables of spindle activity like spindle amplitude or spindle frequency. Because algorithms on spindle detection are different, it is difficult to compare absolute spindle data of different studies. In studies investigating the influence of age on spindle characteristics usually spindles from frontal EEG derivations are analysed, because these spindles are more sensitive to $\mathrm{CNS}$ maturation (Scholle et al. 2007). Comparing our data to other studies analysing spindles from centroparietal derivations in that age group, we find that in our study mean NREM S2 sleep spindle density was quite low (ct. Pivik et al. 1999: > 5/min). We cannot exclude that our visual detection of spindles was less sensitive. Considering stress coping a traitlike personality dimension it would certainly be of high interest to correlate stress coping with the more stable fingerprint approach of sleep EEG, by using a more sophisticated EEG-recording-device in a further study (Finelli et al. 2001; De Gennaro et al. 2005; Tinguely et al. 2006).

\section{Conclusion}

In summary, the present cross-sectional study based on 41 kindergarten children at the age of 5 years showed that sleep spindle activity correlates with cognitive processes related to stress challenges. Therefore, visually detected total number of NREM S2 sleep spindles may reflect children's capability to cope favourably with social stress challenges during wake time. Considering the relevance of coping to learning by everyday challenges, these results fit well with recent discoveries about sleep spindles and memory consolidation during sleep. While stress-elicited HPA-activity reflects 
ego-involvement and active engagement, it seems to depend on cognitive processes, i.e. coping, whether stress challenges lead to increased spindle activity during sleep. Furthermore, well-designed studies are needed to investigate in the relationship between sleep spindles, memory consolidation and stress coping.

\section{Acknowledgements}

We thank R. Hartmann, C. Keppler, L. Kohler, B. Krebs, and P. Walter for their support in data collection. We are deeply grateful to Vladimir Djurdjevic and Marielle Köenig for technical support and data elaboration. We also thank the CortLab of the University of Trier (Germany) for analyzing the cortisol samples. Finally, we thank all participating children, parents, and kindergarten teachers for their support of the study.

\section{Statement of Interest}

The work was supported by the Swiss National Science Foundation SNF No. 32-68193.02 (MH) as well as SNF No. 32-66778.01 (KvK). The SNF had no further role in the analysis and interpretation of the data, the writing of the report, or the decision to submit the paper for publication. All authors declare no conflict of interest.

\section{References}

Blair C, Granger D, Peters Razza R. 2005. Cortisol reactivity is positively related to executive function in preschool children attending head start. Child Dev 76:554-567.

Bódizs R, Kis T, Lázár AS, Havrán L, Rigó P, Clemens $Z$, et al. 2005 . Prediction of general mental ability based on neural oscillation measures of sleep. J Sleep Res 14:285-292.

Born J. 2010. Slow-wave sleep and the consolidation of long-term memory. World J Biol Psychiatry 11(Suppl 1):16-21.

Brand S, Lehtinen A, Hatzinger M, Holsboer-Trachsler E. 2010. Comparison of sleep EEG profiles of patients suffering from restless legs syndrome, restless legs syndrome and depressive symptoms, and major depressive disorders. Neuropsychobiology $61: 41-48$.

Buske-Kirschbaum A, Jobst $S$, Wustmans $S$, Kirschbaum C, RauhW, Hellhammer D. 1997. Attenuated free cortisol response to psychosocial stress in children with atopic dermatitis. Psychosom Med 59:419-426.

Buzsáki G. 1996. The hippocampo-neocortical dialogue. Cereb Cortex 6:81-92.

Compas BE. 2006. Psychobiological processes of stress and coping: implications for resilience in children and adolescents comments on the papers of Romeo \& McEwen and Fisher et al. Ann NY Acad Sci 1094:226-234.

Compas BE, Connor-Smith J, Jaser SS. 2004. Temperament, stress reactivity, and coping: implications for depression in childhood and adolescence. J Clin Child Adolesc Psychol $33: 21-31$
Connor-Smith JK, Compas BE, Wadsworth $\mathrm{ME}$, Thoms en $\mathrm{AH}$, Saltzman H. 2000. Responses to stress in adolescence: measurement of coping and involuntary stress responses. J Consult Clin Psychol 68:976-992.

De Gennaro L, Ferrara M. 2003. Sleep spindles: an overview. Sleep Med Rev 7:423-440.

De Gennaro L, Ferrara $M$, Vecchio $F$, Curcio $G$, Bertini $M$. 2005. An electroencephalographic fingerprint of human sleep. Neuroimage 26:114-122.

Diekelmann S; Born 1. 2010. The memory function of sleep. Nature Rev Neurosci 11:114-126.

Dressendörfer RA, Kirschbaum C, Rohde W, Stahl F, Strasburger CJ. 1992. Synthesis of a cortisol-biotin conjugate and evaluation as a tracer in an immunoassay for salivary cortisol measurement. J Steroid Biochem Mol Biol 43:683-692.

Dunn LM, Dunn ES. 1981. Peabody picture vocabulary test revised (PPV-R). Manual for form $L$ and $M$. Circle Pins (MN): American Guidance Service.

El-Sheikh M, Buckhalt JA, Keller PS, Granger DA. 2008. Children's objective and subjective sleep disruptions: links with afternoon cortisol levels. Health Psychol 27:26-33.

Finelli LA, Achermann P, Borbély AA. 2001. Individual 'fingerprints' in human sleep EEG topography. Neuropsychopharmacology 25(5 Suppl):S57-62.

Fischer S, Wilhelm I, Born J. 2007. Developmental differences in sleep's role for implicit off-line learning: comparing children with adults. J Cogn Neurosci 19:214-227.

Flinn MV, England BG. 1997. Social economics of childhood glucocorticoid stress response and health. Am J Phys Anthropol 102:33-53.

Fogel SM, Smith CT. 2006. Learning-dependent changes in sleep spindles and Stage 2 sleep. J Sleep Res 15:250-255.

Fogel SM, Nader R, Cote KA, Smith CT. 2007. Sleep spindles and learning potential. Behav Neurosci 121:1-10.

Forsythe AI, Keenan TA, Organick EI, Stenberg W. 1969. Computer science: a first course. New York: Wiley.

Gais S, Mölle $M$, Helms K, Born J. 2002. Learning-dependent increases in sleep spindle density. J Neurosci 22:6830-6834.

Genzel L, Dresler $M$, Wehrle R, Grözinger $M$, Steiger A. 2009. Slow wave sleep and REM sleep awakenings do not affect sleep dependent memory consolidation. Sleep 32:302-310.

Grigg-Damberger $M$, Gozal D, Marcus CL, Quan SF, Rosen CL, Chervin RD, et al. 2007. The visual scoring of sleep and arousal in infants and children. J Clin Sleep Med 3:201-240.

Harris AH, Reeder R, Hyun JK. 2009. Common statistical and research design problems in manuscripts submitted to highimpact psychiatry journals: what editors and reviewers want authors to know. J Psychiatr Res 43:1231-1234.

Hasselmo ME. 2006. The role of acetylcholine in learning and memory. Curr Opin Neurobiol 16:710-715.

Hatzinger $M$, Hemmeter UM, Baumann $K$, Brand $S$, Holsboer-Trachsler E. 2002. The combined DEX-CRH test in treatment course and long-term outcome of major depression. J Psychiatr Res 36:287-297.

Hatzinger $M$, Hemmeter $U M$, Brand $S$, Ising $M$, Holsboer-Trachsler E. 2004. Electroencephalographic sleep profiles in treatment course and long-term outcome of major depression: association with DEX/CRH-test response. J Psychiatr Res 38:453-465.

Hatzinger $M$, Brand $S$, Perren $S$, von Wyl $A$, von Klitzing $\mathrm{K}$, Holsboer-Trachsler E. 2007. Hypothalamicpituitary-adrenocortical (HPA) activity in kindergarten children: importance of gender and associations with behavioral/ emotional difficulties. J Psychiatr Res 41:861-870.

Hatzinger $M$, Brand $S$, Perren $S$, Stadelmann $S$, von Wyl A, von Klitzing $K$, et al. 2008. Electroencephalographic sleep profiles and hypothalamic-pituitary-adrenocortical (HPA)-activity in kindergarten children: Early indication of poor sleep quality 
associated with increased cortisol secretion. J Psychiatr Res 42:532-543.

Hatzinger $M$, Brand $S$, Perren $S$, Stadelmann $S$, von Wyl A, von Klitzing $\mathrm{K}$, et al. 2010. Sleep actigraphy pattern and behavioral/emotional difficulties in kindergarten children: association with hypothalamic-pituitary-adrenocortical (HPA) activity. J Psychiatr Res 44:253-261.

Kanaley JA, Weltman JY, Pieper KS, Weltma A, Hartman ML. 2001. Cortisol and growth hormone responses to exercise at different times of day. J Clin Endocrinol Metab 86:2881-2889.

Kirschbaum C, Hellhammer D. 1989. Salivary cortisol in psychobiological research: An overview. Neuropsychobiology 22: 150-169.

Lauer CJ, Riemann D, Wiegand M, Berger M. 1991. From early to late adulthood. Changes in EEG sleep of depressed patients and healthy volunteers. Biol Psychiatry 29:979-993.

Lopez NL, Vazquez DM, Olson SL. 2004. An integrative approach to the neurophysiological substrates of social withdrawal and aggression. Dev Psychopathol 16:69-93.

Marshall L, Born J. 2007. The contribution of sleep to hippocampus-dependent memory consolidation. Trends Cogn Sci 11:442-450.

Measelle JR, Ablow JC, Cowan PA, Cowan CP. 1998. Assessing young children's views of their academic, social, and emotional lives: an evaluation of the self-perception scales of the Berkeley Puppet Interview. Child Dev 69:1556-1576.

Mölle $M$, Born J. 2009. Hippocampus whispering in deep sleep to prefrontal cortex - for good memories? Neuron 61:496 498.

Perren S, von Wyl A, Stadelmann S, Bürgin D, von Klitzing $K$. 2006. Associations between behavioral/emotional difficulties in kindergarten children and the quality of their peer relationships. J Am Acad Child Adolesc Psychiatry 45:867-876.

Pivik RT, Joncas $S$, Busby KA. 1999. Sleep spindles and arousal: the effects of age and sensory stimulation. Sleep Res Online 2:89-100.

Plihal W, Born J. 1997. Effects of early and late nocturnal sleep on declarative and procedural memory. J Cogn Neurosci 9: $534-547$.

Plihal W, Born J. 1999. Memory consolidation in human sleep depends on inhibition of glucocorticoid release. Neuroreport 10:2741-2747.

Rechtschaffen A, Kales A. 1968. A manual of standardized terminology, techniques and scoring system of sleep stages of human subjects. Washington, DC: Public Health Service, US Government Printing Office.

Robinson J, Mantz-Simmons L, Macfie J, Kelsay K. 2002. The MacArthur Narrative Working Group. MacArthur narrative coding manual. Unpublished manuscript. Denver: University of Colorado Health Sciences Center.

Schabus M, Gruber G, Parapatics S, Sauter C, Klösch G, Anderer $P$, et al. 2004. Sleep spindles and their significance for declarative memory consolidation. Sleep 27:1479-1485.

Schabus M, Hödimoser K, Gruber G, Sauter C, Anderer P, Klösch $G$, et al. 2006. Sleep spindle-related activity in the human EEG and its relation to general cognitive and learning abilities. Eur J Neurosci 23:1738-1746.

Schabus $M$, Hoedlmoser $K$, Pecherstorfer $T$, Anderer $P$, Gruber G, Parapatics S, et al. 2008. Interindividual sleep spindle differences and their relation to learning-related enhancements. Brain Res 1191:127-135.

Scholle S, Zwacka G, Scholle HC. 2007. Sleep spindle evolution from infancy to adolescence. Clin Neurophysiol 118: $1525-1531$.

Shinomiya S, Nagata K, Takahashi K, Masumura T. 1999. Development of sleep spindles in young children and adolescents. Clin Electroencephalogr 30:39-43.

Shirtcliff EA, Granger DA, Booth A, Johnson D. 2005. Low salivary cortisol levels and externalizing behavior problems in youth. Dev Psychopathol 17:167-184.

Stadelmann S. 2006. Konfliktbasierte Spielnarrative von Kindergartenkindern: Zusammenhänge mit der Verhaltensregulation und dem familiären Umfeld [Conflict-based playing narratives of kindergarten children. Associations between regulation of behavior and family environment]. Freiburg: Dissertation an der Wirtschafts- und Verhaltenswissenschaftlichen Fakultät der Albert-Ludwigs-Universität Freiburg.

Steiger A. 2002. Sleep and the hypothalamo-pituitary-adrenocortical system. Sleep Med Rev 6:125-138.

Steiger A. 2007. Neurochemical regulation of sleep. J Psychiatr Res 41:537-552.

Stickgold R, 2005. Sleep-dependent memory consolidation. Nature 437:1272-1278.

Stickgold R, Walker MP. 2007. Sleep-dependent memory consolidation and reconsolidation. Sleep Med 8:331-343.

Tinguely G, Finelli LA, Landolt HP, Borbély AA, Achermann P. 2006. Functional EEG topography in sleep and waking: state-dependent and state-independent features. Neuroimage 32:283-292.

Tunn S, Möllmann H, Barth J, Derendorf H, Krieg M. 1992. Simultaneous measurement of cortisol in serum and saliva after different forms of cortisol administration. Clin Chem 38: 1491-1494.

van Goozen SH, Matthys W, Cohen-Kettenis PT, Gispen-de Wied C, Wiegant VM, van Engeland H. 1998. Salivary cortisol and cardiovascular activity during stress in oppositionaldefiant disorder boys and normal controls. Biol Psychiatry 43:531-539.

Von Klitzing K, Kelsay K, Emde RN. 2003. The structure of 5-year-old children's play narratives within the MacArthur Story Stem Methodology. In: Emde RN, Wolf DP, Oppenheim $D$, editors. Revealing the inner world of young children. The MacArthur Story Stem Battery and parent-child narratives. New York: Oxford University Press. p. 106-128.

Wagner U, Born J. 2008. Memory consolidation during sleep: interactive effects of sleep stages and HPA regulation. Stress $11: 28-41$.

Weitzman ED, Fukushima D, Nogeire C, Roffwarg H, GallagherTF, Hellman L. 1971. Twenty-four hour pattern of the episodic secretion of cortisol in normal subjects. J Clin Endocrinol Metab 33:14-22.

Wilhelm I, Diekelmann S, Born J. 2008. Sleep in children improves memory performance on declarative but not procedural tasks. Learn Mem 15:373-377.

Ziegert DI, Kistner JA, Castro R, Robertson B. 2001. Longitudinal study of young children's responses to challenging achievement situations. Child Dev 72:609-624. 